\title{
Analysing the Outcome of a Learning Process Conducted Within the System ALS_CORR[LP]
}

\author{
https://doi.org/10.3991/ijet.v12i03.6377 \\ Nihad El Ghouch \\ Faculty of Sciences and Technologies, UAE, Tangier, Morocco \\ nihad_elghouch@hotmail.fr \\ En-naimi El Mokhtar \\ Faculty of Sciences and Technologies, UAE, Tangier, Morocco \\ ennaimi@gmail.com \\ Yassine Zaoui Seghroucheni \\ Faculty of Sciences, Tetuan, Morocco \\ yassinezaouiseghroucheni@gmail.com
}

\begin{abstract}
This paper presents the results of an experiment, conducted on a sample of computer science students, using the adaptive learning system called ALS_CORR $[\mathrm{LP}]^{1}$. Indeed, unlike the traditional LMS, adaptive learning systems provide a personalized learning experience based on specific objectives, prerequisites and learning styles, generating thereby a specific learning path. However, the main issue resides in the fact that they assume that the generated learning path is supposedly the leading one, which is far from being true, as we can always detect some failure cases during the evaluation phase. In this paper we will conduct a learning experiment using the system ALS_CORR[LP], which has the ability to correct the generated learning path by recommending the most relevant learning objects, and update the learner model based on a calculation of similarity in behavior between the struggling learner and the succeeding ones. We will later analyze the results of behavior tracking within the system.
\end{abstract}

Keywords-Learning style, Learning path, Experiment, Adaptive learning system.

\section{Introduction}

Adaptive learning systems make content dynamic and interactive, placing the learner at the centre of the learning experience. The techniques used to provide adaptability have been summarized by [1] into several techniques and methods. The authors of [2] made it clear that those systems operate according to two strategies:

${ }^{1}$ Adaptive Learning System with a CORRection of Learning Path 
We will use some explicit methods, [3], [4], [5] and [6], to gather information about the learner's preferences, learning-wise, using mainly e-questionnaires, where they can express their tendencies. We will use implicit methods, [7], [8] and [9], to monitor how the student interacts with the learning system, grouping data on the interaction of the learner with the learning objects. Despite the adaptation provided in both cases, failure is an undeniable reality in the learning process. In the case of adaptive learning systems, failure amounts to a detection of error in the learner's preferences, or sometimes in the relevance of the learning objects.

In this paper, we will present the results of an experiment conducted on bachelor of computer science students, using the current MERISE method as a subject of learning on the adaptive learning platform called ALS_CORR[LP] [10]. The rest of this paper is organized as follows:

In Section 2, we will begin with a presentation of the characteristics of the ALS_CORR [LP] system, exposing its learning scenario and architecture. Later, in Section 3, we will focus on the implementation of the experiment. In Section 4, we will analyze the outcome of the experiment and provide some comparisons, using graphical representations of the results. Then, in Section 5, we will provide a graphical representation of the learning style combination of the related students. In Section 6, we will display some statistics about the versions of the learning objects. Finally, some conclusions will be drawn in the last section.

\section{The Style Adaptive Learning System}

With the runaway success of e-learning systems in their abilities to meet specific needs, a new kind of system is booming, namely the adaptive learning system. We are currently witnessing a transition from the one-size-fits-all systems [11] to new ones, which are more interested in a personalization of the learning process. In fact, adaptive learning systems are an important class of the e-learning systems; they usually customize the learning process according to the needs, prerequisites, objectives, etc... of each learner, creating thereby a specific learning path. The problem with these systems is that they assume the generated learning path as systematically the leading one, which is not necessarily the case, as we can still detect several negative results during the evaluation phase.

The ALS_CORR [LP] system presented in this paper has the ability to correct the learning path, using a recommendation system operating in two modes: to recommend the most relevant versions of each learning object by calculating the similarities in behavior between the struggling learner and those who have achieved positive results, provided they have the same initial profile, and recommending later their learning objects. The second mode is to update the initial profile in case the behavior observed in the system does not correspond to the original profile generated initially. 


\subsection{System architecture}

The ALS_CORR[LP] architecture [12] operates using the components displayed in the following figure:

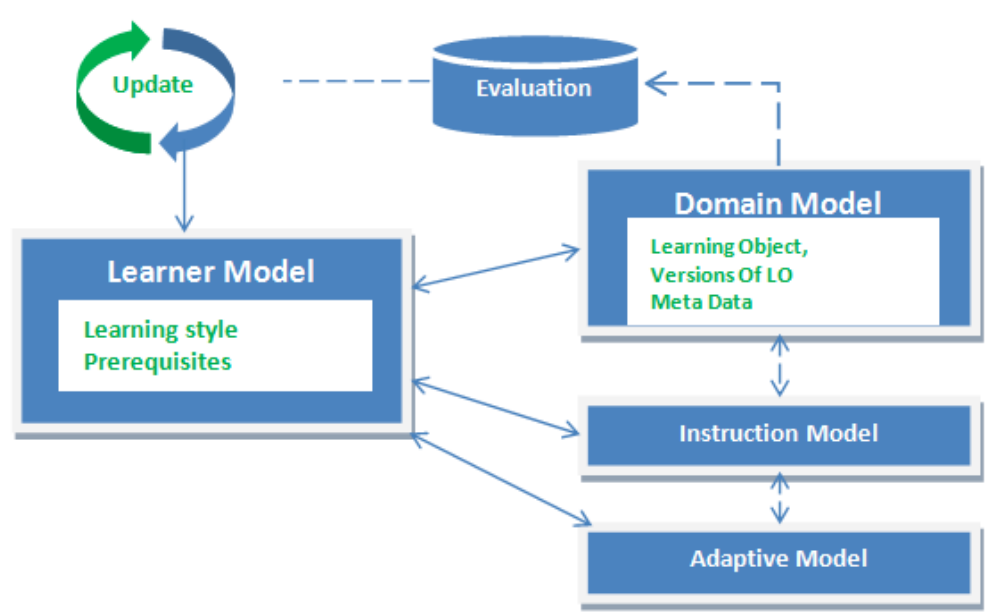

Fig. 1. System Architecture

According to the figure, there are five major components that run the system, namely: the Learner model, the Domain model, the Instruction model, the Adaptive model and the Evaluation phase.

- The Learner model: it is based on Felder-Silverman's test to detect the learning style of each learner and on the prerequisite test.

- The Domain model: it is composed of the learning objects, designed according to the SCORM Standards [13], multiple versions of the same learning object and finally the Content Metadata as defined by IEEE Learning object Metadata.

- The Instruction model: it is the pedagogical model, responsible for designing the learning object.

- The Adaptive model: it enables the system to assign the learning objects according to the characteristics of the learner's profile.

- The Evaluation: it represents a critical part [14] in this adaptive learning system, as it remains the only way to correct the learning path in case it appears not to be the leading one.

\subsection{Learning scenario}

For a first-timer, the learner must respond to a prerequisite test and fill in the test of Felder-Silverman's learning style model known as FSLSM [15], in order to determine the initial profile. The system then assigns an appropriate version [16] of the specific course, based on the preferences expressed in the early profile. In case where the 
generated learning path is not the leading one, which is translated, assessment-wise, by a negative result during the evaluation phase, the system recommends the most relevant versions of the same course based on the calculation of similarity in behavior between the struggling learner and those who have succeeded during the evaluation phase.

\section{Conducting the experiment}

The aim is to use the system ALS_CORR [LP] to conduct an experiment on a sample of bachelor of computer science students, in order to study their behavior inside the adaptive learning system, and more particularly to check the relevance adaptation performed in the system, by comparing the learning model and the observed behavior.

As part of the Design Method module, where the MERISE method of learning takes place, an experiment was conducted on a sample of 53 students of computer engineering degree at the Faculty of Science and Technology of Tangier. To ensure students' engagement with regard to the process, the final score for each learner was calculated, including the results of the evaluation conducted within the system. In the next section, we will present the results relating to the calculation of Felder-Silverman learning styles within the system.

A first analysis of the results registered in the system reveals the following distribution of profiles according to the Felder-Silverman test:

Table 1. Felder-Silverman test result within the system ALS_CORR[LP]

\begin{tabular}{|c|c|c|c|c|c|}
\hline Learner's Number & Sensing & Intuitive & Visual & Verbal & Test Duration (Sec) \\
\hline 1 & 27,27 & 72,73 & 45,45 & 54,55 & 36 \\
\hline 2 & 81,82 & 18,18 & 90,91 & 9,09 & 28 \\
\hline 3 & 100,00 & 0,00 & 90,91 & 9,09 & 11 \\
\hline 4 & 9,09 & 90,91 & 9,09 & 90,91 & 10 \\
\hline 5 & 18,18 & 81,82 & 18,18 & 81,82 & 21 \\
\hline 6 & 54,55 & 45,45 & 63,64 & 36,36 & 261 \\
\hline 7 & 9,09 & 90,91 & 72,73 & 27,27 & 130 \\
\hline 8 & 90,91 & 9,09 & 54,55 & 45,45 & 471 \\
\hline 9 & 90,91 & 9,09 & 63,64 & 36,36 & 447 \\
\hline 10 & 54,55 & 45,45 & 54,55 & 45,45 & 173 \\
\hline 11 & 54,55 & 45,45 & 72,73 & 27,27 & 89176 \\
\hline 12 & 54,55 & 45,45 & 81,82 & 18,18 & 2669 \\
\hline 13 & 100,00 & 0,00 & 100,00 & 0,00 & 25 \\
\hline 14 & 63,64 & 36,36 & 54,55 & 45,45 & 124 \\
\hline 15 & 72,73 & 27,27 & 27,27 & 72,73 & 105 \\
\hline 16 & 27,27 & 72,73 & 90,91 & 9,09 & 503 \\
\hline 17 & 18,18 & 81,82 & 90,91 & 9,09 & 537 \\
\hline 18 & 81,82 & 18,18 & 54,55 & 45,45 & 253 \\
\hline & & & & & \\
\hline & & & & & \\
\hline & & & & & \\
\hline
\end{tabular}


Paper-Analysing the Outcome of a Learning Process Conducted Within the System ALS_CORR[LP]

\begin{tabular}{|c|c|c|c|c|c|}
\hline Learner's Number & Sensing & Intuitive & Visual & Verbal & Test Duration (Sec) \\
\hline 19 & 90,91 & 9,09 & 63,64 & 36,36 & 121 \\
\hline 20 & 81,82 & 18,18 & 63,64 & 36,36 & 81 \\
\hline 21 & 72,73 & 27,27 & 63,64 & 36,36 & 123 \\
\hline 22 & 54,55 & 45,45 & 36,36 & 63,64 & 594 \\
\hline 23 & 54,55 & 45,45 & 54,55 & 45,45 & 340 \\
\hline 24 & 27,27 & 72,73 & 36,36 & 63,64 & 1129 \\
\hline 25 & 45,45 & 54,55 & 72,73 & 27,27 & 275 \\
\hline 26 & 36,36 & 63,64 & 36,36 & 63,64 & 156 \\
\hline 27 & 54,55 & 45,45 & 54,55 & 45,45 & 126 \\
\hline 28 & 9,09 & 90,91 & 45,45 & 54,55 & 246 \\
\hline 29 & 81,82 & 18,18 & 45,45 & 54,55 & 84 \\
\hline 30 & 100,00 & 0,00 & 90,91 & 9,09 & 10 \\
\hline 31 & 9,09 & 90,91 & 9,09 & 90,91 & 7 \\
\hline 32 & 9,09 & 72,73 & 45,45 & 45,45 & 1122 \\
\hline 33 & 54,55 & 45,45 & 63,64 & 36,36 & 175 \\
\hline 34 & 90,91 & 9,09 & 90,91 & 9,09 & 160 \\
\hline 35 & 100,00 & 0,00 & 90,91 & 9,09 & 13 \\
\hline 36 & 36,36 & 63,64 & 54,55 & 45,45 & 155 \\
\hline 37 & 54,55 & 45,45 & 63,64 & 36,36 & 128 \\
\hline 38 & 45,45 & 54,55 & 81,82 & 18,18 & 110 \\
\hline 39 & 100,00 & 0,00 & 90,91 & 9,09 & 86 \\
\hline 40 & 63,64 & 36,36 & 72,73 & 27,27 & 201 \\
\hline 41 & 81,82 & 18,18 & 72,73 & 27,27 & 298 \\
\hline 42 & 63,64 & 36,36 & 100,00 & 0,00 & 136 \\
\hline 43 & 81,82 & 18,18 & 90,91 & 9,09 & 263 \\
\hline 44 & 54,55 & 45,45 & 72,73 & 27,27 & 211 \\
\hline 45 & 72,73 & 27,27 & 72,73 & 27,27 & 217 \\
\hline 46 & 36,36 & 54,55 & 63,64 & 36,36 & 435 \\
\hline 47 & 36,36 & 45,45 & 54,55 & 27,27 & 168 \\
\hline 48 & 63,64 & 36,36 & 36,36 & 63,64 & 133 \\
\hline 49 & 81,82 & 18,18 & 54,55 & 45,45 & 69 \\
\hline 50 & 72,73 & 18,18 & 45,45 & 54,55 & 113 \\
\hline 51 & 54,55 & 45,45 & 81,82 & 18,18 & 168 \\
\hline 52 & 9,09 & 90,91 & 18,18 & 81,82 & 3 \\
\hline 53 & 27,27 & 27,27 & 27,27 & 18,18 & 7 \\
\hline
\end{tabular}

Let us start with an analysis of each dimension of Felder-Silverman's profile used in the system. The following graph shows a comparison of the perception dimension. 


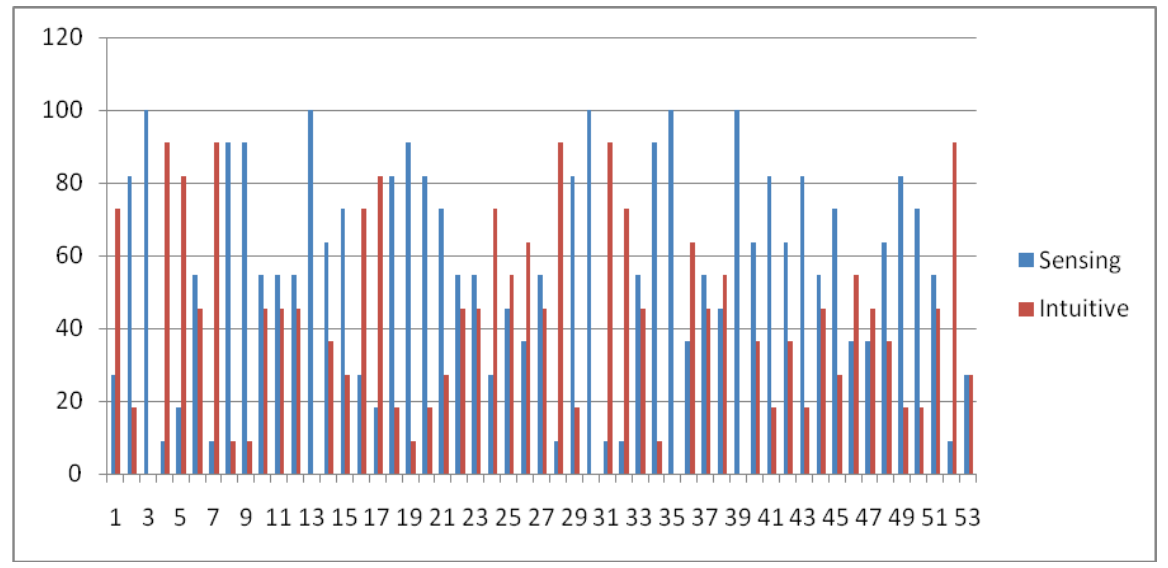

Fig. 2. "Perception" dimension analysis

The following graph relates to the analysis of the "input" dimension of FelderSilverman's profile.

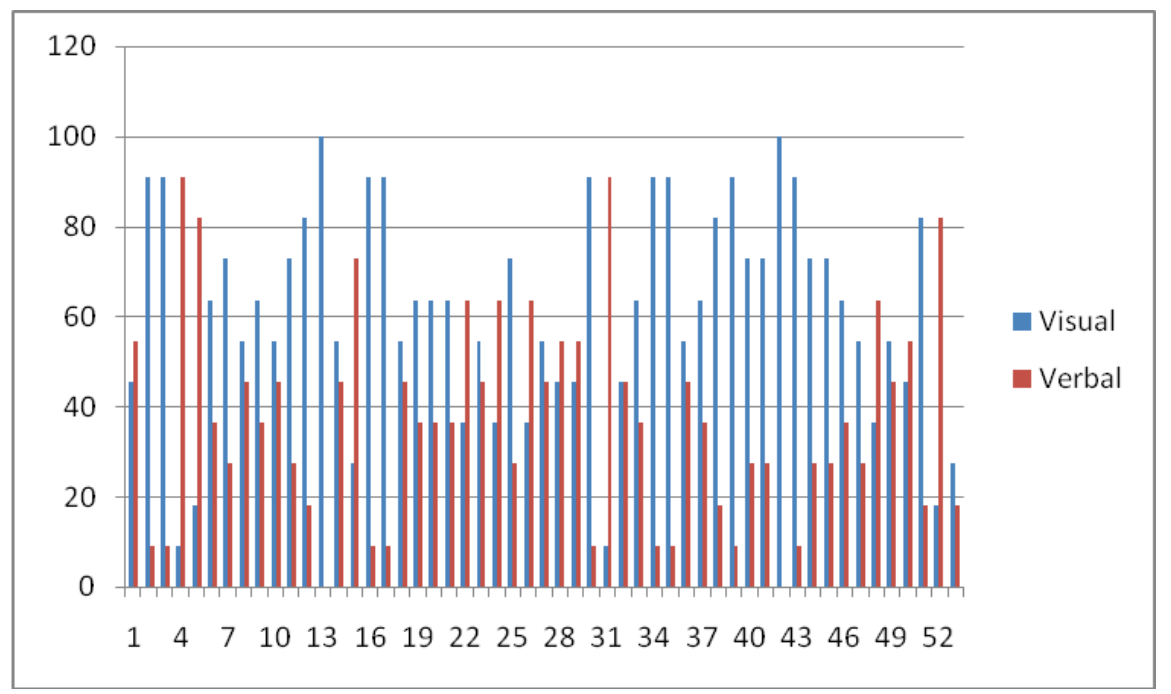

Fig. 3. "Input" dimension analysis

According to the two preceding paragraphs, "Sensing" and "Visual" styles are the most dominant among students' learning preferences. Thus, we can point out that there are four possible combinations, depending on Felder-Silverman's profiles:

- $\{$ Sensing, Visual $\}$

- $\{$ Sensing, Verbal\}

- $\{$ Intuitive, Visual $\}$

- $\{$ Intuitive, Verbal\} 
Here are the results regarding the styles $\{$ Sensing, Visual $\}$; in other words, these students prefer to work with examples and facts of visual kind (videos, diagrams, images, illustrations).

Table 2. $\{$ Sensing, Visual $\}$ Combination

\begin{tabular}{|c|c|c|c|c|c|}
\hline Learner's Number & Sensing & Intuitive & Visual & Verbal & Test Duration (Sec) \\
\hline 2 & 81,82 & 18,18 & 90,91 & 9,09 & 28 \\
\hline 3 & 100,00 & 0,00 & 90,91 & 9,09 & 11 \\
\hline 6 & 54,55 & 45,45 & 63,64 & 36,36 & 261 \\
\hline 8 & 90,91 & 9,09 & 54,55 & 45,45 & 471 \\
\hline 9 & 90,91 & 9,09 & 63,64 & 36,36 & 447 \\
\hline 10 & 54,55 & 45,45 & 54,55 & 45,45 & 173 \\
\hline 11 & 54,55 & 45,45 & 72,73 & 27,27 & 89176 \\
\hline 12 & 54,55 & 45,45 & 81,82 & 18,18 & 2669 \\
\hline 13 & 100,00 & 0,00 & 100,00 & 0,00 & 25 \\
\hline 14 & 63,64 & 36,36 & 54,55 & 45,45 & 124 \\
\hline 18 & 81,82 & 18,18 & 54,55 & 45,45 & 253 \\
\hline 19 & 90,91 & 9,09 & 63,64 & 36,36 & 121 \\
\hline 20 & 81,82 & 18,18 & 63,64 & 36,36 & 81 \\
\hline 21 & 72,73 & 27,27 & 63,64 & 36,36 & 123 \\
\hline 23 & 54,55 & 45,45 & 54,55 & 45,45 & 340 \\
\hline 27 & 54,55 & 45,45 & 54,55 & 45,45 & 126 \\
\hline 30 & 100,00 & 0,00 & 90,91 & 9,09 & 10 \\
\hline 33 & 54,55 & 45,45 & 63,64 & 36,36 & 175 \\
\hline 34 & 90,91 & 9,09 & 90,91 & 9,09 & 160 \\
\hline 35 & 100,00 & 0,00 & 90,91 & 9,09 & 13 \\
\hline 37 & 54,55 & 45,45 & 63,64 & 36,36 & 128 \\
\hline 39 & 100,00 & 0,00 & 90,91 & 9,09 & 86 \\
\hline 40 & 63,64 & 36,36 & 72,73 & 27,27 & 201 \\
\hline 41 & 81,82 & 18,18 & 72,73 & 27,27 & 298 \\
\hline 42 & 63,64 & 36,36 & 100,00 & 0,00 & 136 \\
\hline 43 & 81,82 & 18,18 & 90,91 & 9,09 & 263 \\
\hline 44 & 54,55 & 45,45 & 72,73 & 27,27 & 211 \\
\hline 45 & 72,73 & 27,27 & 72,73 & 27,27 & 217 \\
\hline 49 & 81,82 & 18,18 & 54,55 & 45,45 & 69 \\
\hline 51 & 54,55 & 45,45 & 81,82 & 18,18 & 168 \\
\hline 52 & 9,09 & 90,91 & 18,18 & 81,82 & 3 \\
\hline
\end{tabular}

Here is the second combination of possible learning styles, in which there are learners who tend to favor learning with examples and facts, but of textual nature. 
Table 3. $\{$ Sensing, Verbal $\}$ Combination

\begin{tabular}{|c|c|c|c|c|c|}
\hline Learner's Number & Sensing & Intuitive & Visual & Verbal & Test Duration (Sec) \\
\hline 15 & 72,73 & 27,27 & 27,27 & 72,73 & 105 \\
\hline 22 & 54,55 & 45,45 & 36,36 & 63,64 & 594 \\
\hline 29 & 81,82 & 18,18 & 45,45 & 54,55 & 84 \\
\hline 48 & 63,64 & 36,36 & 36,36 & 63,64 & 133 \\
\hline 50 & 72,73 & 18,18 & 45,45 & 54,55 & 113 \\
\hline
\end{tabular}

This category of learners prefers learning through definitions and algorithms, presented as videos, images, illustrations, etc.

Table 4. $\{$ Intuitive, Visual $\}$ Combination

\begin{tabular}{|c|c|c|c|c|c|}
\hline Learner Number & Sensing & Intuitive & Visual & Verbal & Test Duration Sec \\
\hline 7 & 9,09 & 90,91 & 72,73 & 27,27 & 130 \\
\hline 8 & 90,91 & 9,09 & 54,55 & 45,45 & 471 \\
\hline 16 & 27,27 & 72,73 & 90,91 & 9,09 & 503 \\
\hline 17 & 18,18 & 81,82 & 90,91 & 9,09 & 537 \\
\hline 25 & 45,45 & 54,55 & 72,73 & 27,27 & 275 \\
\hline 36 & 36,36 & 63,64 & 54,55 & 45,45 & 155 \\
\hline 38 & 45,45 & 54,55 & 81,82 & 18,18 & 110 \\
\hline 46 & 36,36 & 54,55 & 63,64 & 36,36 & 435 \\
\hline
\end{tabular}

The latest combination of learning styles focuses on learners who enjoy learning with definitions and algorithms of a textual nature.

Table 5. \{ Intuitive, Verbal\} Combination

\begin{tabular}{|c|c|c|c|c|c|}
\hline Learner's Number & Sensing & Intuitive & Visual & Verbal & Test Duration (Sec) \\
\hline 1 & 27,27 & 72,73 & 45,45 & 54,55 & 36 \\
\hline 4 & 9,09 & 90,91 & 9,09 & 90,91 & 10 \\
\hline 5 & 18,18 & 81,82 & 18,18 & 81,82 & 21 \\
\hline 24 & 27,27 & 72,73 & 36,36 & 63,64 & 1129 \\
\hline 26 & 36,36 & 63,64 & 36,36 & 63,64 & 156 \\
\hline 28 & 9,09 & 90,91 & 45,45 & 54,55 & 246 \\
\hline 31 & 9,09 & 90,91 & 9,09 & 90,91 & 7 \\
\hline 8 & 90,91 & 9,09 & 54,55 & 45,45 & 471 \\
\hline
\end{tabular}

\section{Graphical representation of the learning style combination}

It is obvious that the combination of learning styles \{Sensing, Visual\} is the dominant combination in the system. In fact, students in this class promote learning with visual resources (videos, images, illustrations...) while learning objects are in the form of examples and explanations. 


\section{FSLSM Profiles}

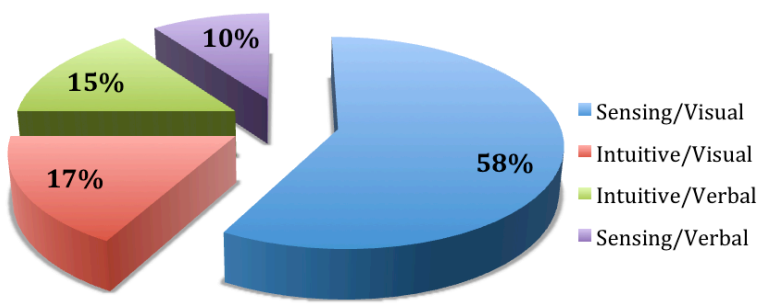

Fig. 4. Distribution of learning style combinations

Indeed, the graph showing the distribution obtained about learning styles based on combinations shows that approximately two thirds of students have the combination \{ Sensing, Visual \}, while the remaining third is divided almost equally among the other combinations of learning styles.

\section{$5 \quad$ Statistics About the Learning Object Versions}

ALS_CORR [LP] system allows for several statistics about the learning objects and their versions, depending especially on their nature, type and resources. It also allows pointing out the most visited versions of the course.

Below, we will present an overview of the statistics obtained by the system regarding the learning of the MERISE design method.

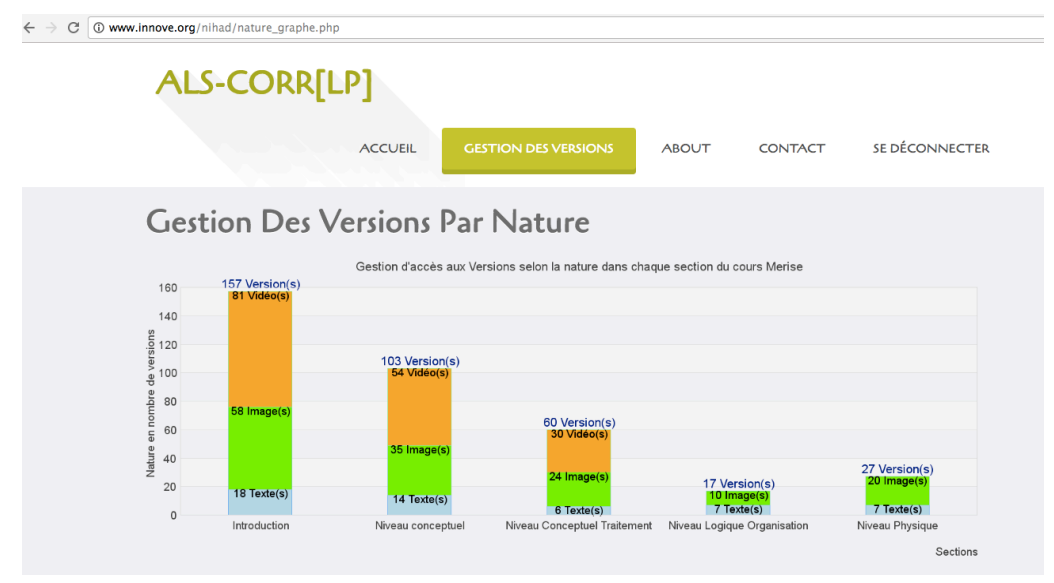

Fig. 5. Statistics related to the nature of the versions of the learning objects

The graph above shows that the video version of the course introduction is the most viewed of all sections. It is also noteworthy that the video versions of the other sections are the most visited too.

Here is an example of statistics on the most visited versions in seconds. 


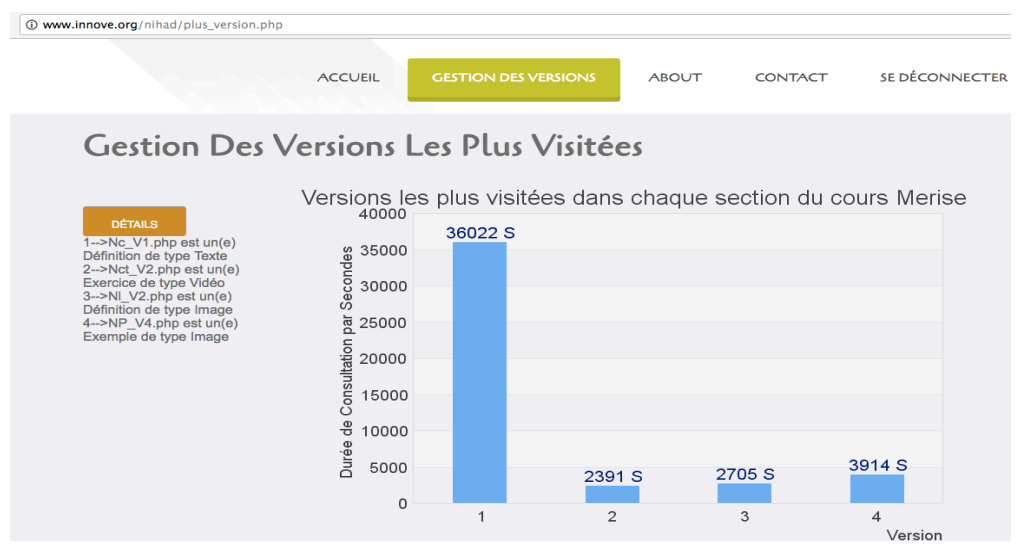

Fig. 6. Statistics about the most visited versions (s)

\section{Evaluation Results}

The main objective of the evaluation is to make sure that learners did achieve the educational goals set at the start, and to get a summary of, or judgment on, the teaching strategies adopted. This phase is crucial in the system because it provides it with the necessary information, on which it can rely to reassess the relevance of the calculation made in the Domain model.

Here is the result of the assessment regarding the learning of MERISE method by the computer science students.

\section{Evaluation result}

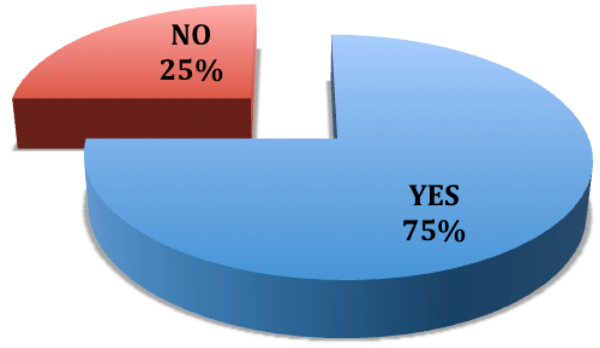

Fig. 7. Evaluation results

The figure above shows that $75 \%$ were able to overcome this phase successfully, against $25 \%$ who experienced difficulties during this phase. The former high percentage could certainly be explained by the adaptation that took place within the system. Nevertheless, we can still see cases of failure during the evaluation phase. Therefore, we will put the focus on how the system has performed during this phase. 


\section{$7 \quad$ Learning Path Correction}

We will look at the case of students with negative results during the evaluation phase. We take as an example the case of a student "C-L". Here is the result of the similarity analysis by Bravais Pearson Formula, based on the behavior of other students who have passed this stage successfully.

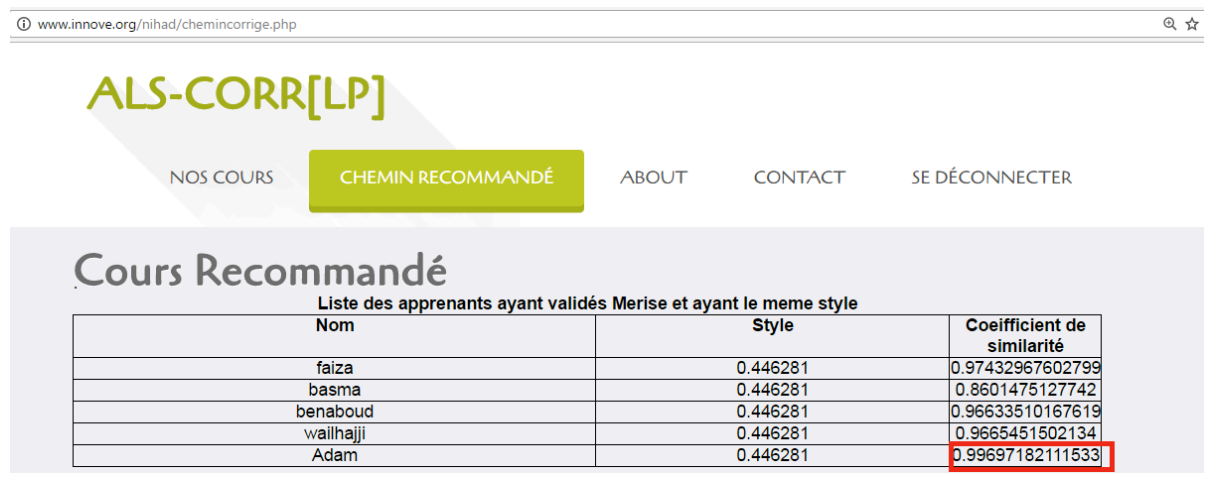

Fig. 8. Similarity calculation based on Bravais-Pearson formula between the learner named " $\{\mathrm{C}-\mathrm{L}\}$ " and the other learners having the same learning style

The maximum similarity in behavior is up to 0.896257856337 . The system recommends the learner to follow the same course, but with versions of the learner with whom this similarity has proved to be at maximum. He can have access to these new versions as shown in the following figure:

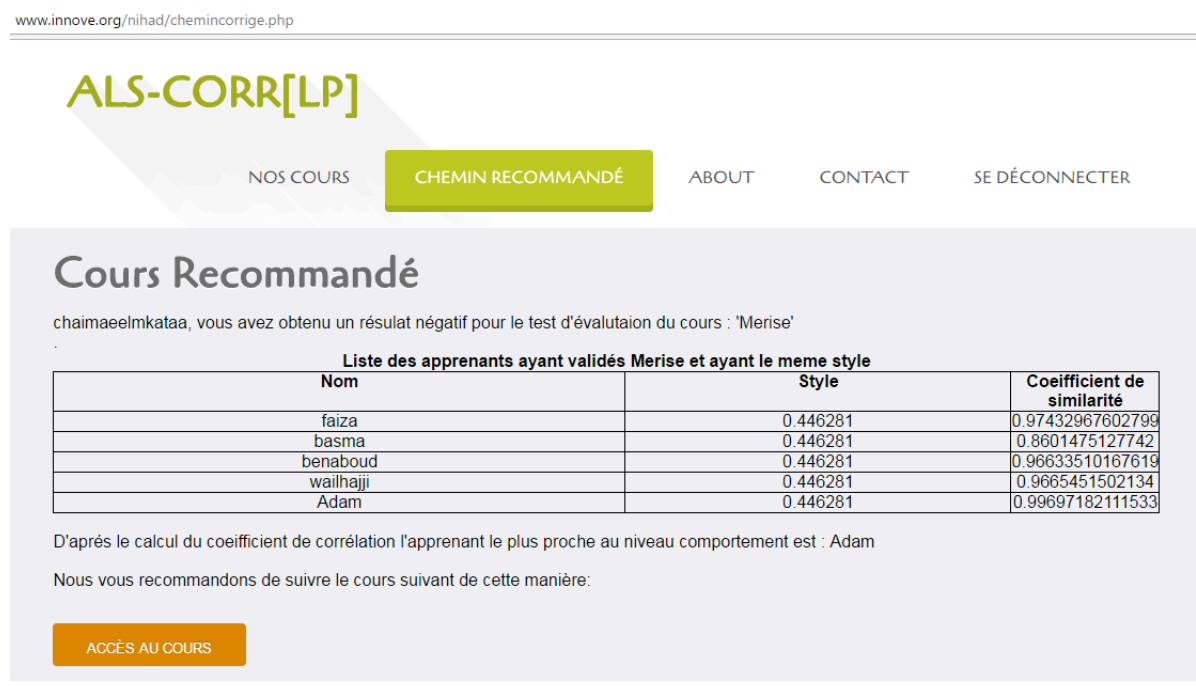

Fig. 9. Recommendation of new versions of courses based on the similarity results 
Access to the course is possible once again, but this time while offering the versions that match the behavior observed in the learner "C-L" in the system. Here is an example of the displayed versions:

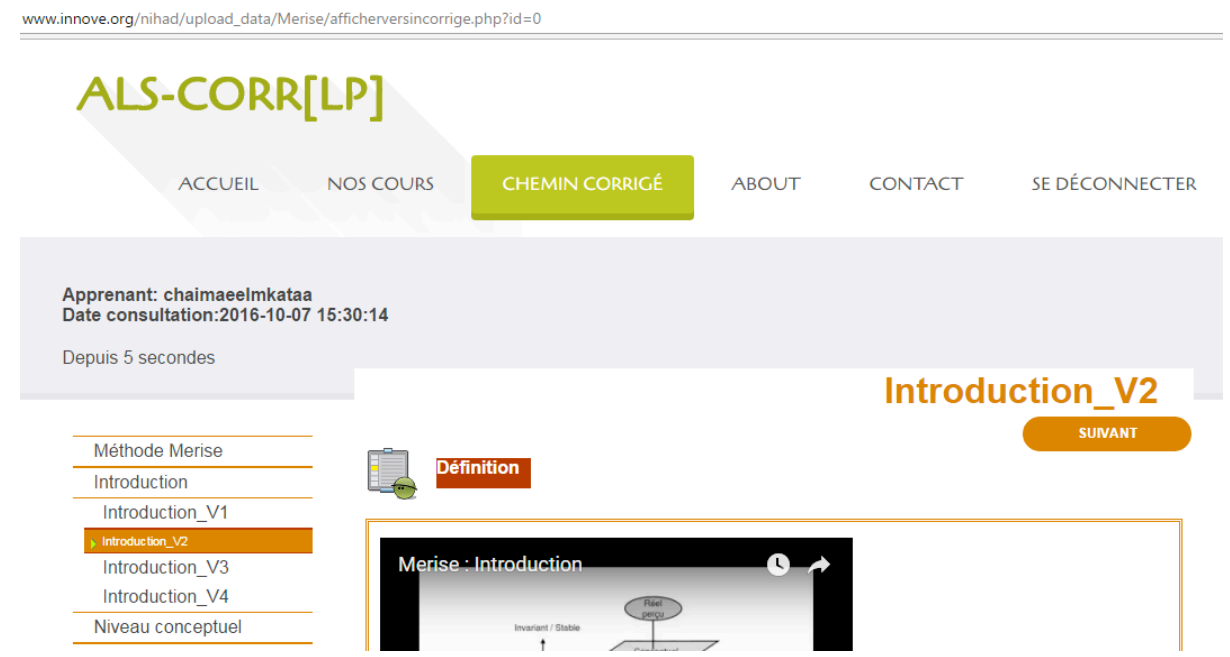

Fig. 10. Access to the new versions of the MERISE method course

\section{Conclusion and Perspectives}

The current paper presents the results of a learning process that took place within the system ALS_CORR[LP]. 53 students took a course in the MERISE method. The results obtained during the evaluation phase were very encouraging, to the extent that they showed that $75 \%$ were able to validate the learning process, while the remaining $25 \%$ have been experiencing some difficulties during the evaluation phase. Students who had difficulties during the learning phase could follow the same course, but with new versions whose relevance has been deduced through the calculation of similarity in behavior between learners in difficulties and other learners with the same original learning style, and who managed to overcome successfully the evaluation phase.

The next step is to adapt our adaptive learning system AIS_CORR[LP] to mobile learning, by studying the constraints of the latter which has experienced a very important development.

\section{$9 \quad$ References}

[1] Seghroucheni, Y. Z., Mohajir, B. E. E. "Exploitation of the recommendation systems in the calculation of the learning path". 5th International Conference on Information and Communication Systems (ICICS), ISO 690, April, 2014. https://doi.org/10.1109/iacs.2014.6841985 
[2] Seghroucheni, Y. Z., \& Mohajir, B. E. E.. "Exploitation of the recommendation systems in the calculation of the learning path", 5th International Conference on Information and Communication Systems (ICICS), ISO 690, April, 2014. https://doi.org/10.1109/iacs.2014.6841985

[3] Tzouveli, P., Mylonas, P., \& Kollias, S. “An intelligent e-learning system based on learner profiling and learning resources adaptation”, Computers \& Education, 51(1), 224-238, 2008. https://doi.org/10.1016/j.compedu.2007.05.005

[4] Bontchev, B., \& Vassileva, D. Courseware. "Adaptation to Learning Styles and Knowledge Leve". Edited by Anderson Silva, Elvis Pontes, 1, 2012.

[5] Graf, S., Viola, S. R., Leo, T., \& Kinshuk. "In-depth analysis of the Felder- Silverman learning style dimensions". Journal of Research on Technology in Education, 40(1), 79-93, 2007. https://doi.org/10.1080/15391523.2007.10782498

[6] Guerrero- Roldán ، an, A. E., \& Alfonso, J. M. "Adaptive learning paths for improving lifelong learning experiences". Service Oriented Approaches and Lifelong Competence Development Infrastructures, 137, 2007.

[7] Carchiolo, V., Longheu, A., Malgeri, M., \& Mangioni, G. "An architecture to support adaptive e-learning". International Journal of Computer Science and Network Security, 7(1), 166-178, 2007.

[8] Bousbia, N., Reba, I., Labat, J. M., \& Balla, A.. "Analyzing the record Relationship between Learning Styles and Navigation Behavior in Web-Based Educational System", Knowledge Management ELearning. An International Journal, 2(4), 400, 2009.

[9] Schiaffino, S., Garcia, P., Amandi, A. eTeacher. "Providing personalized assistance to elearning students". Computers Education, 51(4), 1744-1754, 2008. https://doi.org/10.1016/j.compedu.2008.05.008

[10] Seghroucheni, Y. Z., Achhab, M. A. "Implementation of an Adaptive Learning System that Include Correction of Learning Path Based on the Differentiated Pedagogy and the Bayesian Network". International Journal of Recent Contributions from Engineering, Science IT (iJES), 3(2), 27-31, 2015. https://doi.org/10.3991/ijes.v3i2.4389

[11] Brusilovsky, P., Kobsa, A., Vassileva, J. (Eds.). "Adaptive hypertext and hypermedia". Kluwer Academic, 1998.

[12] Seghroucheni, Y. Z., Achhab, M. A. A. "Bayesian Network Based on the Differentiated Pedagogy to Generate Learning Object According to FSLSM". International Journal of Recent Contributions from Engineering, Science IT (iJES), 3(2), 21-26, 2015. https://doi.org/10.3991/ijes.v3i2.4363

[13] Nihad Elghouch, El Mokhtar En-Naimi, Yassine Zaoui Seghroucheni, Badr Eddine El Mohajir, Mohammed Al Achhab. "An application to index the didactic resources in an adaptive learning system (ALS)". 5th International Conference on Information Communication Technology and Accessibility ICTA (pp. 1-3). IEEE, December 2015. https://doi.org/10.1109/icta.2015.7426893

[14] Nihad Elghouch, El Mokhtar En-Naimi, Yassine Zaoui Seghroucheni, Badr Eddine El Mohajir, Mohammed Al Achhab. "Highlighting the evaluation gaits in an adaptive learning system". The International Conference on Communication, Management and Information Technology ICCMIT Cosenza-Italy, 26-29 April 2016.

[15] Felder, R. M., Silverman, L.K. "Learning and teaching styles in engineering education". Engineering education, 78(7), 674-681, 1988.

[16] Seghroucheni, Y. Z., Mohammed, A., El Mohajir, B. E. "An Approach to Create Multiple Versions of the Same Learning Object".International Journal of Emerging Technologies in Learning, 9(5), 2014. https://doi.org/10.3991/ijet.v9i5.3762 


\section{Authors}

Nihad EL GHOUCH is a PhD student in Faculty of Sciences and Technologies (FST) of Tangier, department of Computer Science, the University of Abdelmalek Essaadi, FST of Tangier - Morocco. Enrolled in the laboratory LIST (Laboratoire d'Informatique, Systèmes et Télécommunications).

El Mokhtar EN-NAIMI is a Professor in Faculty of Sciences and Technologies (FST) of Tangier, department of Computer Science. He is a member of the Laboratory LIST (Laboratoire d'Informatique, Systèmes et Télécommunications), the University of Abdelmalek Essaadi, FST of Tangier - Morocco. Mr. EN-NAIMI is also a Head of Computer Science Department at the FST of Tangier. In addition, he is an associate member of the ISCN-Institute of Complex Systems in Normandy, the University of The Havre - France, since 2009.

Yassine Zaoui Seghrouchen is a PhD student in The Faculty of Sciences, Tetuan, University of Abdelmalek Essaadi, FST of Tangier - Morocco.

Article submitted 28 October 2016. Published as resubmitted by the authors 23 January 2017. 